\title{
A REVIEW OF CHRYSANTHEMUM, THE EASTERN QUEEN IN TRADITIONAL CHINESE MEDICINE WITH HEALING POWER IN MODERN PHARMACEUTICAL SCIENCES
}

\author{
SHAHRAJABIAN, M. H. ${ }^{1,2 \#}-$ SUN, W. ${ }^{1,2 \#}-$ ZANDI, P. ${ }^{3}-$ CHENG, Q. ${ }^{1,2^{*}}$ \\ ${ }^{1}$ Biotechnology Research Institute, Chinese Academy of Agricultural Sciences, Beijing 100081, \\ China \\ ${ }^{2}$ Nitrogen Fixation Laboratory, Qi Institute, Building C4, No. 555 Chuangye, Jiaxing 314000, \\ Zhejiang, China \\ ${ }^{3}$ Institute of Environment and Sustainability, Development in Agriculture, Chinese Academy of \\ Agricultural Sciences, Beijing 100081, China \\ ${ }^{\#}$ These authors equally contributed to this paper. \\ *Corresponding author \\ e-mail: chengqi@caas.cn \\ (Received 12 $2^{\text {th }}$ Jun 2019; accepted $28^{\text {th }}$ Aug 2019)
}

\begin{abstract}
Chrysanthemum is famous as the Queen of the East grown mainly in China and Japan. Chrysanthemum is the second most important plant in both the ornamental industry and traditional Chinese medicine. The most important chemical extracts of Chrysanthemum include flavonoids, betaine, choline and vitamin B1. Thirteen important compounds of chrysanthemum flowers are acacetin-7-0-betaD-glucopyranoside, luteolin, luteolin-7-O-beta-D-glucopyranoside, acaciin, acacetin 7-0-(6/1-0-alpha-Lrhamnopyranosyl)-beta-sophoroside, 3-0-caffeoylquinic acid, syringaresinol 0-beta-D-glucopyranoside, 5,7-dihydroxychromone, uracil, p-gydroxybenzoic acid, 4-0-beta-D-glucopyranosyloxybenzoic acid, boscialin and blumenol A. The most outstanding health benefits of chrysanthemum tea are easing stress and anxiety, improving cardiovascular health, protect against oxidative damage, inhibit inflammation, support healthy immune function, improve eye health and lower risk for osteoporosis. In traditional Chinese medicine, chrysanthemum is a cold herb which helps dispel pathogenic heat, helps the liver, improves eyesight and aids detoxification. To conclude, treatment with natural Chinese herbal medicine especially chrysanthemum, non-synthetic drug is recommended for an organic life.
\end{abstract}

Keywords: health benefits, Asian medicinal science, western pharmaceutical science, herbal medicine, silk road

\section{Introduction}

Traditional Chinese medicine is a system of medicine based on acupuncture, acupressure, Chinese herbs, cupping, diet and moxibustion (Soleymani and Shahrajabian, 2012, 2018; Ogbaji et al., 2018; Shahrajabian et al., 2018, 2019a, b, c). Traditional medicine refers to health practices, knowledge, approaches and beliefs incorporating plants and herbs based on both ancient and modern pharmaceutical science (Sharajabian et al., 2019d, e, f, 2020). Chrysanthemum with high ornamental value is a one of the ten most popular traditional flowers in China and one of the most popular cut flowers in the world (Sun et al., 2011; Wang et al., 2014; Chung et al., 2018). Wang et al. (2014) stated that there are more than 20,000 chrysanthemum cultivars in the world and about 7,000 cultivars in China. The objective of this review is survey on some important modern and ancient pharmaceutical sciences of chrysanthemum. 


\section{Materials and methods}

All relevant papers in the English language of researchers from different countries were collected. The keywords of chrysanthemum, traditional Chinese medicine, traditional Asian medicine, modern pharmaceutical science, health benefits and western medicine were searched in Google Scholar, Scopus, Research Gate and PubMed.

\section{Results and discussion}

\section{Chrysanthemum occurrence and cultivation}

In Chinese culture, ancient Chinese scholars consider chrysanthemum as a symbol of nobility and integrity and long prized by ordinary people for its medicinal properties. Teixeria (2003) mentioned that, chrysanthemum is the world's second most economically important floricultural crop, following rose. In China, it is found most often in Zhejiang, Anhui, Henan and Sichuan provinces. Imtiaz et al. (2019) also noted that chrysanthemum is native to China and was first cultivated as a flowering herb back in the 15th century BC, and it was believed that this plant had the power of life. Chrysanthemum is famous as Queen of the East, and also known as autumn flower (Saicharan et al., 2017). In traditional Chinese medicine, chrysanthemum provides mildly cold energy, and it has special affinity to the energy channels that lead to the lungs, liver, spleen and kidneys. Chrysanthemum tea is an herbal infusion made from the dried flowers of the chrysanthemum plant in ancient China, and it was used as an herbal remedy in traditional Chinese medicine (TCM) as early as 1500 B.C. Its petals consumed in the form of a salad has the perception of causing longevity. Chrysanthemum commonly called as gul-e-daudi or golden flower autumn queen has been cultivated for more than 2000 years ago in Iran. It belongs to the family of Asteraceae, and it behaves both as an annual as well as perennial flowering crop (Kalia, 2015). It is the world's second most important floricultural crop only after Rose (Kalia, 2015). The National Chrysanthemum Society of Britain lists over 600 cultivars of this plant (Datta, 2013). Klie et al. (2014) has shown that chrysanthemum is a segmental allohexaploid with an ambiguous pattern of inheritance. Chrysanthemums require welldrained soil and full sunlight to grow and successfully bloom. Plants grown with less light will become weak, spindly and produce few flowers. Chrysanthemums are shallow rooted and do best if planted high, which means that frequent watering may be necessary during times of high heat and little rainfall. The best defense against adverse weather conditions is to provide good drainage so that water does not accumulate around the plants and promote ice formation. Cicek Atikmen et al. (2014) found that $12.5 \%$ fresh mushroom compost and $25 \%$ exhausted mushroom compost were the best ratios for cultivation of chrysanthemum. Chrysanthemum white rust (CWR) is one of the primary diseases on chrysanthemum which has been considered as a quarantine disease in many countries (Dong et al., 2018). List of some chrysanthemum varieties is presented in Table 1. Qualitative characters of 20 genotypes of chrysanthemum is shown in Table 2. Genotypes of C. morifolium is shown in Table 3. Performance of chrysanthemum genotypes for vegetative characters is presented in Table 4. Length of flowering, diameter of flowering stem, diameter of flower bud, diameter of most open inflorescence, plant height, and number of inflorescences per pot of chrysanthemum is presented in Table 5. Phytochemical characteristics evaluated in chrysanthemum plants (average of three cultivars) with and without application Si is shown in Table 6. Chukki 
et al. (2018) indicated that Chrysanthemum indicum flower has the potential for Congo red dye reduction from aqueous solution. A number of disease plague chrysanthemus, are Septoria Leaf Spot, Powdery Mildew and virus diseases such as mosaic and stunt or virus-like diseases such as aster yellows. A number of insects such as Aphids, Caterpillars, Leafhoppers, Leaminers, Plant Bugs and Spider Mites may hurt the crops. Chrysanthemums cultivars reveal amazing colours, shapes types of inflorescence and a varied growth (Carvalho-Zanao et al., 2012). Plants are generally raised through suckers and terminal cuttings. It has been said that micro propagation to be very efficient technique for the fast and disease free raising of Chrysanthemum plants (Kalia, 2015). Cojocariu et al. (2018) noted that knowing the particular architecture can improve the correct application of chrysanthemum culture technologies as properly positioning of the crops into the appropriate fertility plots, adequate application of fertilizers, and mechanical maintenance of chrysanthemum crops in order to protect the root system of the plants. Yasemin et al. (2017) reported that flower diameters, disc florets, number of flowers, shoot height, root collar thickness, root and shoot fresh weights were negatively affected in 150 and $200 \mathrm{mM} \mathrm{NaCl}$ treatments. Liu and Xiao (2018) reported that fourteen compounds were isolated and identified as stigmata-4-ene-3-one (1),

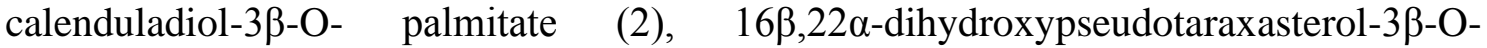

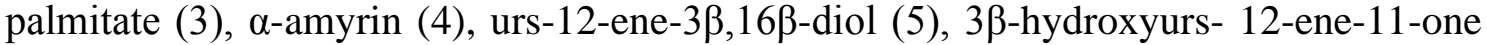
(6), arnidiol (7), maniladiol (8), 3 $\beta$-hydroxyolean-12-ene-11-one (9), luteolin (10), apigenin (11), apigenin-7,4'- dimethyl ether (12), genkwanin (13), and 1-linoleic acid glycerate (14). Carvalho-Zanao et al. (2012) reported that silicon has promoted improvements both in quantitative and qualitative aspects when supplied to some ornamental species produced in these conditions. Lee and Van Iersel (2008) found that saline water may be more readily available and can have the added benefit of reduced plant height, which is an important quality characteristic for floriculture crops such as chrysanthemum. The effect of growth substrates over the nutrients of chrysanthemum plant is shown in Table 7. The effect of growth substrates over the nutrients of chrysanthemum plant is presented in Table 8. Germination rate of seeds is shown in Table 9. Kalia (2015) indicated that Chrysanthemum can be multiplied in large scale through micro propagation using right concentration of the auxins. She clearly indicates that the above problems can be minimized by micro propagation of Chrysanthemum.

Table 1. List of some chrysanthemum varieties (Kumar et al., 2014)

\begin{tabular}{c|c}
\hline Cultivars & Cultivars \\
\hline Kanchil & Pusa Anmol \\
Glumohr & Yellow Bangla \\
Shayamal & Sharad Mala \\
Sadwin Yellow & Star White \\
White Andaman & TERI \\
Aparjita & Poornima White \\
Sadbhawna & Waters May \\
Flirt & Beauty \\
Neelima & Jubilee \\
White Prolific & Maghi Orange \\
Ravikiran & Maghi White \\
Birbal Sahni & Maghi Yellow \\
Shukla & Kalvin Orange
\end{tabular}




$$
-13358-
$$

Yellow Charm
Pink Cloud
Kajole
Gaity
Geetanjali
Star Pink
Korean Small
Yellow Star
Ajay
Meghavi
Yellow Gold
Lalpari
Red Gold
Vasantika
Kundan
Kargil
Shanti
Taichen Queen
Star Yellow
Snowball
President Viger
Jayanti
Dolly Orange
Liliput
FDL

Diana

Pankaj

Kalvin Pink

Sonali Tara

Mother Teressa

Pinked White

Gajra

Santa Dine

Red Shringar

Shwet Shringar

White Anemone

Mahatma Gandhi

Raja Orange

Tokyo Soldier

Korean Small

Kalvin Yellow

Greenish White

Ajay

Texas Gold

Yellow Reflex

Golden Yellow

Red D spoon

Annual Chrysanthemum

Annual Chrysanthemum

Table 2. Qualitative characters of 20 genotypes of chrysanthemum (Prakash et al., 2018)

\begin{tabular}{c|c|c|c}
\hline Genotypes & Flower colour & Disc colour & Type of flower \\
\hline Thai Chin Queen & Orange & $*$ & Double \\
Pusa arunoday & Pink & Yellow & Double \\
Yellow charm & Yellow & Yellow & Semi-double \\
Pusa sona & Yellow & Yellow & Semi-double \\
Pusa centenary & Yellow & $*$ & Double \\
Pusa aditya & Yellow with orange center & Orange & Semi-double \\
Sunny & Blood red & $*$ & Double \\
Pusa kesari & Saffron & $*$ & Double \\
Sadbhavana & Dark orange & Yellow & Semi-double \\
Lalith & White & $*$ & Double \\
Ajay & Pink & $*$ & Double \\
Pusa Chitraksha & Deep magenta & Yellow & Semi-double \\
Basanthi & Yellow & Yellow & Semi-double \\
Ramlal dada & Yellow & $*$ & Single \\
Haldighati & Dark yellow & $*$ & Double \\
Star white & White & $*$ & Double \\
Lal pari & Red & Yellow & Semi-double \\
Jaya & White & $*$ & Double \\
Ajay orange & Orange & $*$ & Double \\
Lilyput & Yellow & $*$ & Double \\
\hline
\end{tabular}




$$
-13359 \text { - }
$$

Table 3. Genotypes of C. morifolium (Kaur et al., 2018)

\begin{tabular}{c|c|c|c|c|c}
\hline Plant tag no. & Genotypes name & Color & Plant tag no. & Genotypes name & Color \\
\hline V1 & Aparajita & Yellow & V9 & Celtic & Green \\
V2 & Fortune & White & V10 & Paiwer-W & White \\
V3 & Anastasia & White & V11 & HF-164 & Yellow Purple \\
V4 & Charlia & Purple Yellow & V12 & Paladov Dark & Orange \\
V5 & Vanilla Sorbet & Cream & V13 & Tocovar-6 & Red \\
V6 & Paladov Sunny & Yellow & V14 & Papaya & Orange \\
V7 & White Double & White & V15 & Cologne & White \\
V8 & Braca Splendid & Magenta & & & \\
\hline
\end{tabular}

Table 4. Performance of chrysanthemum genotypes for vegetative characters (Kaur et al., 2018)

\begin{tabular}{|c|c|c|c|c|c|c|c|}
\hline Population No. & Genotypes & $\begin{array}{l}\text { Plant spread } \\
(\mathbf{c m})\end{array}$ & $\begin{array}{l}\text { No. of branches } \\
\text { per plant }\end{array}$ & $\begin{array}{c}\text { No. of leaves } \\
\text { per plant }\end{array}$ & $\begin{array}{c}\text { No. of flowers } \\
\text { per stem }\end{array}$ & $\begin{array}{l}\text { No. of flowers } \\
\text { per cut flower }\end{array}$ & $\begin{array}{c}\text { Flower size } \\
(\mathrm{cm})\end{array}$ \\
\hline V1 & Aparajita & 26.06 & 13.66 & 156.33 & 4.73 & 55.44 & 3.73 \\
\hline $\mathrm{V} 2$ & Fortune & 31.86 & 15.06 & 140.93 & 5.46 & 60.34 & 5.73 \\
\hline V3 & Anastasia & 17.86 & 7.53 & 51.00 & 4.33 & 27.33 & 5.43 \\
\hline V4 & Charlia & 22.40 & 18.46 & 252.73 & 4.46 & 52.38 & 3.28 \\
\hline V5 & Vanilla Sorbet & 12.13 & 10.06 & 73.93 & 2.60 & 23.80 & 3.92 \\
\hline V6 & Paladov Sunny & 16.26 & 11.26 & 82.13 & 2.86 & 31.74 & 2.59 \\
\hline V7 & White Double & 23.80 & 13.26 & 156.00 & 4.26 & 46.16 & 5.71 \\
\hline V8 & Braca Splendid & 21.26 & 9.46 & 75.40 & 3.53 & 34.13 & 5.15 \\
\hline V9 & Celtic & 21.00 & 6.20 & 57.46 & 4.26 & 26.11 & 3.04 \\
\hline V10 & Paiwer-W & 23.66 & 9.33 & 98.33 & 4.60 & 43.93 & 5.11 \\
\hline V11 & HF-164 & 20.93 & 8.06 & 72.00 & 2.93 & 21.74 & 5.13 \\
\hline V12 & Paladov Dark & 13.66 & 6.00 & 34.66 & 2.20 & 20.53 & 4.58 \\
\hline V13 & Tocovar-6 & 23.00 & 12.00 & 145.40 & 3.13 & 38.87 & 5.05 \\
\hline V14 & Papaya & 19.40 & 10.13 & 99.46 & 3.06 & 38.88 & 5.21 \\
\hline V15 & Cologne & 18.73 & 9.40 & 77.86 & 2.13 & 19.78 & 4.93 \\
\hline $\begin{array}{l}\text { C.D. at } 5 \% \text { level } \\
\text { of significance }\end{array}$ & & 3.63 & 3.29 & 5.58 & 1.56 & 1.99 & 0.17 \\
\hline
\end{tabular}

Table 5. Length of flowering (LFS), diameter of flowering stem (DFS), diameter of flower bud (DFB), diameter of most open inflorescence (DI), plant height (PH), and number of inflorescences per pot (NI) of chrysanthemum cultivar (Carvalho-Zanao et al., 2012)

\begin{tabular}{c|c|c|c|c|c|c}
\hline Cultivar & $\begin{array}{c}\text { PH } \\
(\mathbf{c m})\end{array}$ & $\begin{array}{c}\text { DI } \\
(\mathbf{c m})\end{array}$ & $\begin{array}{c}\text { LFS } \\
(\mathbf{c m})\end{array}$ & $\begin{array}{c}\text { DFS } \\
(\mathbf{c m})\end{array}$ & $\begin{array}{c}\text { DFB } \\
(\mathbf{c m})\end{array}$ & $\begin{array}{c}\text { NI } \\
(\mathbf{u n i t} / \mathbf{p o t})\end{array}$ \\
\hline Coral Charm & $55.35 \mathrm{a}$ & $5.15 \mathrm{c}$ & $44.94 \mathrm{a}$ & $44.94 \mathrm{a}$ & $0.99 \mathrm{a}$ & $22.50 \mathrm{c}$ \\
White Reagan & $56.31 \mathrm{a}$ & $6.87 \mathrm{~b}$ & $45.09 \mathrm{a}$ & $45.09 \mathrm{a}$ & $1.01 \mathrm{a}$ & $29.17 \mathrm{a}$ \\
Indianapolis & $48.67 \mathrm{~b}$ & $7.81 \mathrm{a}$ & $41.10 \mathrm{a}$ & $41.10 \mathrm{a}$ & $1.16 \mathrm{a}$ & $24.50 \mathrm{~b}$ \\
\hline CV $(\%)$ & 6.42 & 3.85 & 6.51 & 6.51 & 6.34 & 15.15 \\
\hline
\end{tabular}




$$
-13360 \text { - }
$$

Table 6. Phytochemical characteristics evaluated in chrysanthemum plants as a function of the application of Si (average of three cultivars) (Carvalho-Zanao et al., 2012)

\begin{tabular}{|c|c|c|c|}
\hline Variables & Without Si & With Si & $\mathrm{CV}(\%)$ \\
\hline Length of flowering stem (cm) & $39.99 \mathrm{a}$ & $39.22 \mathrm{a}$ & 7.42 \\
\hline Diameter of flowering stem $(\mathrm{cm})$ & $0.38 \mathrm{a}$ & $0.37 \mathrm{a}$ & 6.51 \\
\hline Diameter of flower bud (cm) & $1.00 \mathrm{a}$ & $1.03 \mathrm{a}$ & 6.34 \\
\hline Diameter of the most open inflorescence $(\mathrm{cm})$ & $6.65 \mathrm{a}$ & $6.58 \mathrm{a}$ & 3.85 \\
\hline Height of plant $(\mathrm{cm})$ & $48.73 \mathrm{a}$ & $49.45 \mathrm{a}$ & 6.42 \\
\hline Number of inflorescences per pot & $25.11 \mathrm{a}$ & $25.67 \mathrm{a}$ & 15.15 \\
\hline Production of root dry matter $(\mathrm{g})$ & $2.88 \mathrm{a}$ & $2.87 \mathrm{a}$ & 20.60 \\
\hline Production of leaf dry matter (g) & $7.36 \mathrm{a}$ & $6.86 \mathrm{a}$ & 14.49 \\
\hline Production of stems dry matter (g) & $11.36 \mathrm{a}$ & $11.50 \mathrm{a}$ & 15.57 \\
\hline Production of inflorescence dry matter (g) & $7.62 \mathrm{a}$ & $7.49 \mathrm{a}$ & 19.58 \\
\hline Production of dry matter of the shoots (g) & $26.34 \mathrm{a}$ & $25.84 \mathrm{a}$ & 11.16 \\
\hline Cycle (days) & $88.54 \mathrm{a}$ & $88.50 \mathrm{a}$ & 7.55 \\
\hline Shelf life (days) & $26.89 \mathrm{a}$ & $27.00 \mathrm{a}$ & 6.21 \\
\hline
\end{tabular}

Averages followed by distinct letters different significantly among each other by Turkey's test, $\mathrm{p}<0.05$

Table 7. The effect of growth substrates over the nutrients of chrysanthemum plant (Cicek Atikmen et al., 2014)

\begin{tabular}{|c|c|c|c|c|c|}
\hline Growth substrates & $\begin{array}{c}\text { Total } \mathbf{N} \\
(\%)\end{array}$ & $\begin{array}{c}\text { Total P } \\
(\%)\end{array}$ & $\begin{array}{c}\text { Total K } \\
(\%)\end{array}$ & $\begin{array}{c}\text { Total Na } \\
\text { (ppm) }\end{array}$ & $\begin{array}{c}\text { Total Ca } \\
(\%)\end{array}$ \\
\hline $100 \% \mathrm{P}$ & $4.04^{\mathrm{ns}}$ & $1.07 \mathrm{~A}$ & $6.36^{\mathrm{ns}}$ & $695^{\mathrm{ns}}$ & $1.81 \mathrm{E}$ \\
\hline $12.5 \% \mathrm{FMC}+87.5 \% \mathrm{P}$ & 3.90 & $0.79 \mathrm{CD}$ & 7.14 & 513 & 2.01CDE \\
\hline $25 \% \mathrm{FMC}+75 \% \mathrm{P}$ & 3.84 & $0.87 \mathrm{BC}$ & 6.81 & 581 & $1.86 \mathrm{DE}$ \\
\hline $50 \% \mathrm{FMC}+50 \% \mathrm{P}$ & 3.62 & $0.66 \mathrm{D}$ & 6.55 & 739 & $2.48 \mathrm{AB}$ \\
\hline $12.5 \%$ FMC $+25 \%$ Perlite $+62.5 \% \mathrm{P}$ & 3.98 & $0.80 \mathrm{CD}$ & 6.68 & 705 & 2.07BCD \\
\hline $25 \% \mathrm{FMC}+25 \%$ Perlite $+50 \% \mathrm{P}$ & 3.83 & $0.79 \mathrm{CD}$ & 7.27 & 607 & $2.31 \mathrm{ABC}$ \\
\hline $50 \%$ FMC $+25 \%$ Perlite $+25 \% \mathrm{P}$ & 3.75 & $0.84 \mathrm{BCD}$ & 7.20 & 597 & $2.44 \mathrm{AB}$ \\
\hline $12.5 \% \mathrm{EMC}+87.5 \% \mathrm{P}$ & 3.71 & $0.93 \mathrm{ABC}$ & 6.82 & 784 & $2.25 \mathrm{ABCD}$ \\
\hline $25 \% \mathrm{EMC}+75 \% \mathrm{P}$ & 3.91 & $0.95 \mathrm{ABC}$ & 6.70 & 619 & 2.19ABCDE \\
\hline $50 \% \mathrm{EMC}+50 \% \mathrm{P}$ & 3.81 & $0.93 \mathrm{ABC}$ & 6.83 & 526 & $2.57 \mathrm{~A}$ \\
\hline $12.5 \% \mathrm{EMC}+25 \%$ Perlite $+62.5 \% \mathrm{P}$ & 3.80 & $1.07 \mathrm{~A}$ & 7.05 & 614 & $2.57 \mathrm{~A}$ \\
\hline $25 \%$ EMC $+25 \%$ Perlite $+50 \% \mathrm{P}$ & 3.91 & $1.09 \mathrm{~A}$ & 6.75 & 599 & $2.26 \mathrm{ABCD}$ \\
\hline $50 \%$ EMC $+25 \%$ Perlite $+25 \% \mathrm{P}$ & 3.84 & $1.00 \mathrm{~A}$ & 7.20 & 579 & $2.34 \mathrm{ABC}$ \\
\hline
\end{tabular}

ns: non-significant, $\mathrm{p}<0.01 . \mathrm{P}=$ Peat, $\mathrm{FMC}=$ Fresh $\mathrm{MC}, \mathrm{EMC}=$ Exhausted $\mathrm{MC}$

Table 8. The effect of growth substrates over the nutrients of chrysanthemum plant (Cicek Atikmen et al., 2014)

\begin{tabular}{|c|c|c|c|c|c|}
\hline Growth substrates & $\begin{array}{c}\text { Total Mg } \\
(\%)\end{array}$ & $\begin{array}{c}\text { Total Fe } \\
\text { (ppm) }\end{array}$ & $\begin{array}{c}\text { Total Mn } \\
\text { (ppm) }\end{array}$ & $\begin{array}{c}\text { Total Zn } \\
\text { (ppm) }\end{array}$ & $\begin{array}{c}\text { Total Cu } \\
\text { (ppm) }\end{array}$ \\
\hline $100 \% \mathrm{P}$ & $1.00 \mathrm{~A}$ & $701 \mathrm{~A}$ & $96 \mathrm{BC}$ & 144BC & $17 \mathrm{~A}$ \\
\hline $12.5 \% \mathrm{FMC}+87.5 \% \mathrm{P}$ & $0.86 \mathrm{CD}$ & $405 \mathrm{CD}$ & $88 \mathrm{BC}$ & $166 \mathrm{~B}$ & $14 \mathrm{BC}$ \\
\hline $25 \% \mathrm{FMC}+75 \% \mathrm{P}$ & $0.84 \mathrm{D}$ & $354 \mathrm{D}$ & $103 \mathrm{BC}$ & $100 \mathrm{C}$ & $15 \mathrm{ABC}$ \\
\hline $50 \% \mathrm{FMC}+50 \% \mathrm{P}$ & $0.94 \mathrm{ABC}$ & $306 \mathrm{D}$ & $136 \mathrm{~A}$ & 49D & $14 \mathrm{C}$ \\
\hline $12.5 \%$ FMC $+25 \%$ Perlite $+62.5 \% \mathrm{P}$ & $0.90 \mathrm{BCD}$ & 463BCD & $90 \mathrm{BC}$ & $48 \mathrm{D}$ & $15 \mathrm{ABC}$ \\
\hline $25 \%$ FMC $+25 \%$ Perlite $+50 \% \mathrm{P}$ & $0.92 \mathrm{ABCD}$ & $505 \mathrm{BCD}$ & $86.29 \mathrm{C}$ & $156 \mathrm{~B}$ & $14 \mathrm{C}$ \\
\hline $50 \%$ FMC $+25 \%$ Perlite $+25 \% \mathrm{P}$ & $0.95 \mathrm{ABC}$ & 464BCD & $118 \mathrm{AB}$ & $224 \mathrm{~A}$ & $16 \mathrm{ABC}$ \\
\hline $12.5 \% \mathrm{EMC}+87.5 \% \mathrm{P}$ & $0.95 \mathrm{ABC}$ & $566 \mathrm{ABC}$ & $101 \mathrm{BC}$ & $175 B$ & $16 \mathrm{ABC}$ \\
\hline $25 \% \mathrm{EMC}+75 \% \mathrm{P}$ & $0.92 \mathrm{ABCD}$ & $659 \mathrm{AB}$ & $92 \mathrm{BC}$ & $156 \mathrm{~B}$ & $16 \mathrm{ABC}$ \\
\hline $50 \% \mathrm{EMC}+50 \% \mathrm{P}$ & $0.95 \mathrm{ABC}$ & $702 \mathrm{~A}$ & $81 \mathrm{C}$ & 161B & $14 \mathrm{C}$ \\
\hline $12.5 \%$ EMC $+25 \%$ Perlite $+62.5 \% \mathrm{P}$ & $0.98 \mathrm{AB}$ & 495BCD & $99 \mathrm{BC}$ & $160 \mathrm{~B}$ & $15 \mathrm{ABC}$ \\
\hline $25 \%$ EMC $+25 \%$ Perlite $+50 \% \mathrm{P}$ & $0.97 \mathrm{AB}$ & 466BCD & $81 \mathrm{C}$ & $155 \mathrm{~B}$ & $16 \mathrm{ABC}$ \\
\hline $50 \%$ EMC $+25 \%$ Perlite $+25 \% \mathrm{P}$ & $1.01 \mathrm{~A}$ & 474BCD & $93 \mathrm{BC}$ & $170 \mathrm{~B}$ & $17 \mathrm{AB}$ \\
\hline
\end{tabular}

ns: non-significant, $\mathrm{p}<0.01 . \mathrm{P}=$ Peat, $\mathrm{FMC}=$ Fresh $\mathrm{MC}, \mathrm{EMC}=$ Exhausted $\mathrm{MC}$ 
Table 9. Germination rate of seeds (Wang et al., 2014)

\begin{tabular}{c|c}
\hline Cultivars & Seed germination rate (\%) \\
\hline QX-081 & 0 \\
QX-006 & 0 \\
QX-003 & 0 \\
Q10-33-2 & $66.7 \pm 8.3$ \\
Nannongxiangbin & 0 \\
Nannonghongcheng & $57.1 \pm 6.5$ \\
QX-001 & $66.7 \pm 4.7$ \\
Nannongjinhe & $27.5 \pm 4.5$ \\
Q10-33-1 & $23.9 \pm 4.3$ \\
\hline
\end{tabular}

Values given are mean \pm standard deviation

\section{Medicinal uses and potential health benefits in traditional and modern medicine industry}

In traditional Chinese medicine (TCM), chrysanthemum flowers are plants that belong to the Cool/Acrid herbs that release the exterior category. Herbs which release the exterior aim to treat the early stages of diseases that affect the upper respiratory tract, the eyes, the ears, the nose, the throat or the skin. In some Eastern cultures, chrysanthemums are also a symbol of good luck, wealth, happiness, and longevity. In Chinese medicine, chrysanthemums are a versatile herb called Ju Hua. In Chinese traditional medicine, Ju Hua is considered to be a fragrant, cool, and light herb. Also, it is used for cooling heat in the liver channel, especially when it manifests as dry, red or painful eyes. Yang et al. (2019) discovered that the Chrysanthemum morifolium flower is widely used in China and Japan as a food, beverage, and medicine for many diseases. Chrysanthemum tea is naturally caffeine-free which makes it a great alternative to drinks containing caffeine like black tea and coffee. It has been reported that Chrysanthemum morifolium has many antioxidant activities including resisting fatigue, improving the function of cardiovascular system, and lowering the levels of serum lipid (Wang and Xiao, 2013; Yu et al., 2013). The healing benefits of Chrysanthemum morifolium are closely related to the composition and content of phenolic compounds (Liu et al., 2013), and apigenin-7-O-glucoside is one of the most active phenolic compounds in chrysanthemum flowers (Wang et al., 2018). Sassi et al. (2008) found that chrysanthemum acts as an antibiotic against a variety of pathogens. Marongiu et al. (2009) reported that the extract of a fresh plant can be applied to skin infections. Liang $\mathrm{Yu}$ et al. (2010) and Michalowska and Lema-Ruminska (2018) reported that chrysanthemums have many health-promoting properties used in medicine. Bose et al. (2003) found that chrysanthemum s boiled roots were used as a headache remedy, young sprouts and petals were eaten as salad and leaves were brewed for a festive drink. Chrysanthemum has aesthetic values, antigenotoxic, antioxidative and antimutagenic properties. 13 different types of Chrysanthemums are: Single blooms, Quilled blooms, Spider blooms, Anemone, Pompons, Decorative blooms, Reflex and Incurve blooms, Reflex mums, Brush or Thistle Chrysanthemums, Unclassified, Spoon mums, Cushion mums and Miscellaneous mums. Terpenes concentrations used for sensory analyses is shown in Table 10. Compounds identified from Chrysanthemum morifolium Huangju by UHPLC-Q-TOF-MS is presented in Table 11. Antioxidant activities of apigenin-7- 
O-glucoside, apihenon and glucose is shown in Table 12. The chemical compositions of Chrysanthemum essential oil (CHEO) is presented in Table 13. Essential oil composition of Chrysanthemum cinerariifolium is shown in Table 14. Some health benefits of chrysanthemums are for allergies, hypertension (high blood pressure0, tightening of the chest, anxiety, skin conditions such as boils, vertigo, eyes that are inflamed, headaches, sore throats, colds and tinnitus. The health benefits of chrysanthemum is shown in Table 15. The most important health benefits of chrysanthemum tea is presented in Table 16. Samples and origins of the studies Chrysanthemum morifolium tea from China is shown in Table 17. Common traditional Chinese medicine formulas in which chrysanthemum flowers are used is shown in Table 18.

Table 10. Terpenes concentrations used for sensory analyses (Niu et al., 2018)

\begin{tabular}{c|c}
\hline Terpenes & Concentration (mg/L) \\
\hline$\alpha$-pinene & 3083 \\
Camphene & 2780 \\
$\beta$-pinene & 355 \\
$\beta$-myrcene & 787 \\
$\alpha$-phellandrene & 523 \\
dl-limonene & 8192 \\
Cis-ocimene & 53 \\
$\alpha$-terpinolen & 7566 \\
Caryophyllene & 775 \\
$\beta$-farnesene & 243 \\
Germacrene B & 985 \\
Alcohols & \\
Linalool & 2974 \\
D-fenchyl alcohol & 1178 \\
Eudesmol & 1177 \\
Borneol & 183 \\
Isoborneol & 3065 \\
4-terpineol & 866 \\
\hline
\end{tabular}

Table 11. Compounds identified from Chrysanthemum morifolium Huangju by UHPLC-QTOF-MS (Wang et al., 2018)

\begin{tabular}{|c|c|c|c|c|c|c|}
\hline No. & RT & Formula & {$[\mathrm{M}-\mathrm{H}]^{-}$} & Score & MS/MS & Identification \\
\hline 1 & 4.833 & C16H18O9 & 353.08785 & 97.69 & $\begin{array}{c}191.05644, \\
248.97382, \\
112.98560\end{array}$ & Chlorogenic acid \\
\hline 2 & 11.442 & $\mathrm{C} 21 \mathrm{H} 20 \mathrm{O} 11$ & 447.09376 & 87.93 & 285.03972 & Luteolin-7-O-glucoside \\
\hline 3 & 17.242 & $\mathrm{C} 25 \mathrm{H} 24 \mathrm{O} 11$ & 515.12035 & 95.93 & $\begin{array}{l}353.08736, \\
179.03450, \\
173.04544, \\
135.04501, \\
191.05577\end{array}$ & 3,5-dicafeoylquinic acid \\
\hline 4 & 19.575 & $\mathrm{C} 25 \mathrm{H} 24 \mathrm{O} 12$ & 431.09920 & 95.44 & $\begin{array}{l}268.03810, \\
269.04341 \\
\end{array}$ & Apigenin-7-O-Glucoide \\
\hline 5 & 21.275 & $\mathrm{C} 21 \mathrm{H} 18 \mathrm{O} 11$ & 445.07743 & 79.28 & $\begin{array}{c}269.04514, \\
113.02422 \\
\end{array}$ & Apigenin-7-O-glucuronide \\
\hline 6 & 22.208 & $\mathrm{C} 24 \mathrm{H} 22 \mathrm{O} 14$ & 533.09396 & 94.85 & $\begin{array}{l}489.10472, \\
285.04009 \\
\end{array}$ & $\begin{array}{l}\text { Luteolin-7-O-6"- } \\
\text { malonylglucoside }\end{array}$ \\
\hline 7 & 32.767 & C15H10O5 & 269.04627 & 94.96 & $\begin{array}{c}117.03469, \\
151.00383, \\
149.02439\end{array}$ & Apigenin \\
\hline
\end{tabular}


Table 12. Antioxidant activities of apigenin-7-O-glucoside, apihenon and glucose (Wang et al., 2018)

\begin{tabular}{c|c|c|c}
\hline \pm & ABTS $\left(\mathbf{E C}_{50}\right)$ & DPPH $\left(\mathbf{E C}_{\mathbf{5 0}}\right)$ & FI $\left(\mathbf{E C}_{\mathbf{5 0}}\right)$ \\
\hline Apigenin-7-O-glucoside & $5.49 \pm 0.74^{\mathrm{a}}$ & $/$ & $/$ \\
Apigenin & $0.68 \pm 0.01^{\mathrm{b}}$ & $/$ & $/$ \\
Glucose & $/$ & $/$ & $/$ \\
BHT & $0.17 \pm 0.00^{\mathrm{b}}$ & $0.41 \pm 0.01^{\mathrm{a}}$ & $/$ \\
Ascorbic acid & $0.12 \pm 0.00^{\mathrm{b}}$ & $0.11 \pm 0.00^{\mathrm{b}}$ & $/$ \\
Rutin & $0.52 \pm 0.10^{\mathrm{b}}$ & $0.52 \pm 0.07^{\mathrm{a}}$ & $0.32 \pm 0.03$ \\
EDTA & $/$ & $/$ & $/$ \\
\hline
\end{tabular}

Each value is expressed as the mean \pm standard deviation $(n=3)$. Means with different letters with in a column are significantly different $(\mathrm{p}<0.01)$. ABTS and DPPH, effective concentration at which $50 \%$ of radicals are scavenged $(\mathrm{mg} / \mathrm{mL})$; FI, ferrous ion chelating power; effective concentration at which $50 \%$ of ferrous ions are chelated $(\mathrm{mg} / \mathrm{mL})$. Positive controls were: BHT, ascorbic acid, rutin and EDTA. /, no data obtained from the $\mathrm{EC}_{50}$ model $\mathrm{Y}=100 /\left(1+10^{\wedge}((\operatorname{LogEC} 50-\mathrm{C}) *\right.$ HillSlope $\left.)\right)$

Table 13. The chemical compositions of chrysanthemum essential oil (CHEO) (Lin et al., 2019)

\begin{tabular}{c|c|c|c}
\hline Composition & Proportion (\%) & Composition & Proportion (\%) \\
\hline Borneol & $19.55 \pm 0.031$ & $\alpha$-Curcumene & $1.25 \pm 0.021$ \\
\hline B-Slinene & $16.25 \pm 0.052$ & Eucalyptol & $1.11 \pm 0.013$ \\
\hline Camphor & $13.48 \pm 0.021$ & Pentanoic acid & $1.05 \pm 0.018$ \\
\hline Guaia-3,9-diene & $5.26 \pm 0.019$ & $\begin{array}{c}\text { Butanoic acid, 3-methyl-,1,7,7- } \\
\text { trimethylbicyclo[2.2.1]hept-2-yl ester }\end{array}$ & $1.0 \pm 0.007$ \\
\hline Hexaoxa-cycloocatdecane & $4.16 \pm 0.012$ & $\begin{array}{c}1,4,7,10,13,16- \\
\text { Hexaoxacyclooctadecane }\end{array}$ & $0.92 \pm 0.011$ \\
\hline Cyclopropa-naphthalene & $2.69 \pm 0.015$ & $\begin{array}{c}\text { 3-Cyclohexene-1-methanol, alpha., } \\
\text { alpha., 4-trimethyl }\end{array}$ & $0.92 \pm 0.003$ \\
\hline $\begin{array}{c}\text { 1,4,7,10,13,16- } \\
\text { Hexaoxacycloocladecane }\end{array}$ & $2.33 \pm 0.004$ & 1-Phenyl-2-propanol & $0.63 \pm 0.011$ \\
\hline $\begin{array}{c}\text { 3-ethylidence-1- } \\
\text { methylcyclopentene }\end{array}$ & $1.88 \pm 0.008$ & Octaethylene glycol & $0.56 \pm 0.042$ \\
\hline $\begin{array}{c}\text { Tetramethyl-undeca-2,6,9- } \\
\text { trien-8-one }\end{array}$ & $1.42 \pm 0.014$ & $3,6,9,12,15$-Pentaoxanonadecan-1-ol & $0.27 \pm 0.026$ \\
\hline
\end{tabular}

Table 14. Essential oil composition of Chrysanthemum cinerariifolium (Shrestha et al., 2014)

\begin{tabular}{c|c|c|c|c|c}
\hline RI & Compound & $\mathbf{\%}$ & RI & Compound & \% \\
\hline 809 & 2-Hexanol & 0.8 & 1217 & Trans-Carveol & 0.4 \\
854 & (2E)-Hexenal & 0.4 & 1225 & Neoiso-Dihydrocarveol & 0.3 \\
856 & (3Z)-Hexenol & 2.3 & 1261 & Cis-Chrysanthenyl acetate & 0.5 \\
890 & 2-Hexen-1-ol & 0.3 & 1270 & Unidentified & 1.4 \\
891 & n-Hexanol & 1.0 & 1311 & (Z)-Patchenol & 0.9 \\
941 & a-Pinene & 0.2 & 1315 & Unidentified & 1.9 \\
981 & 1-Octen-3-ol & 1.5 & 1356 & Eugenol & 0.5 \\
992 & Dehydro-1,8-cineole & 0.4 & 1419 & (E)-Caryophyllene & 0.9 \\
994 & 6-Methyl-5-hepten-2-ol & 0.2 & 1458 & (E)- $\beta$-Farnesene & 0.4 \\
996 & 3-Octanol & 0.2 & 1477 & Trans-Cadina-1(6),4-diene & 0.2
\end{tabular}




\begin{tabular}{l|c|c|c|c|c}
1016 & $\alpha$-Terpinene & 0.3 & 1481 & r-Muurolene & 4.6 \\
1024 & p-Cymene & 0.2 & 1484 & ar-Curcumene & 0.3 \\
1028 & Limonene & 0.2 & 1497 & $\alpha$-Zingiberene & 1.0 \\
1030 & 1,8 -Cineole & 2.4 & 1516 & cis-Dihydroagarofuran & 0.7 \\
1032 & Benzyl alcohol & 0.2 & 1525 & $\delta$-Cadinene & 1.7 \\
1043 & Phenylacetaldehyde & 0.4 & 1534 & Italicene Ether & 0.3 \\
1066 & Cis-Sabinene hydrate & 0.4 & 1550 & Unidentified & 1.0 \\
1097 & Trans-Sabinene hydrate & 0.7 & 1552 & Unidentified & 0.9 \\
1100 & Linalool & 0.2 & 1559 & Unidentified & 1.2 \\
1103 & Filifolone & 2.3 & 1565 & (E)-Nerolidol & 0.3 \\
1105 & Hotrienol & 1.1 & 1576 & Germacrene D-4-ol & 1.4 \\
1112 & 2-Phenylethyl alcohol & 1.2 & 1581 & ar-Turmerol & 0.5 \\
1120 & Isophorone & 1.0 & 1583 & Caryophyllene oxide & 2.1 \\
1125 & Chrysanthenone & 7.6 & 1601 & Viridiflorol & 2.2 \\
1138 & trans-Pinocarveol & 3.3 & 1609 & Humulene epoxide II & 0.2 \\
1144 & Camphor & 11.0 & 1628 & 1 -epi-Cubenol & 1.1 \\
1162 & Cis-Chrysanthenol & 4.4 & 1631 & Caryophylla-4(12),8(13)-dien-5 $\alpha$-ol & 0.8 \\
1165 & Borneol & 3.5 & 1633 & Caryophylla-4(12),8(13)-dien-5 $\beta-o l$ & 1.9 \\
1166 & $\delta$-Terpineol & 0.4 & 1642 & $\tau$-Muurolol & 2.7 \\
1173 & Cis-Pinocamphone & 0.2 & 1646 & $\alpha$-Muurolol (= Torreyol) & 0.5 \\
1176 & Terpinen-4-ol & 3.6 & 1651 & $\beta$-Eudesmol & 0.4 \\
1190 & $\alpha$-Terpineol & 1.2 & 1655 & $\alpha$-Cadinol & 4.8 \\
1193 & Methyl salicylate & 1.3 & 1686 & Caryophylla-4(15)5,10(14)-trien-1 $\alpha-o l$ & 0.8 \\
1195 & Myrtenol & 1.0 & 1691 & Shyobunol & 3.4 \\
1202 & Nopol & 0.4 & 1737 & Oplopalone & 1.2 \\
1207 & Verbenone & 0.5 & 1954 & Hexadecanoic acid & 0.3 \\
1208 & Trans-3(10)-Caren-2-ol & 0.2 & 2108 & (E)-Phytol & 0.2 \\
& & & & Total identified & 93.7 \\
\hline
\end{tabular}

Table 15. The health benefits of chrysanthemum

1- Increase the metabolism in the body, which can help people to lose weight, improve circulation, regulate hormone levels, and even improve neurotransmitter activity

2- $\quad$ Prevent certain chronic illnesses, in part because it helps fight free radicals, prevents cellular mutations, and protects body against numerous illnesses which cause by free radicals

3- Improve vision. Chrysanthemum tea can also improve eyesight, and also can protect against diseases such as cataracts, macular, degeneration, neuropathy and even blurry vision

4- It may help to improve bone density and even prevent osteoporosis. This is due to its many naturally occurring minerals, including calcium and magnesium

\begin{tabular}{ll}
\hline $5-$ & It may boost immune system because of its high levels of Vitamin C and A \\
\hline $6-$ & It may help unclog arteries and improve overall heart health \\
\hline $7-$ & It may help alleviate varicose veins \\
\hline $8-$ & $\begin{array}{l}\text { Help ease digestive issues, eliminating a lot of digestive problems and keeping body in less pain with fewer } \\
\text { stomach problems }\end{array}$ \\
\hline $9-$ & It may help alleviate dryness and itchiness in the eyes \\
\hline $10-$ & Rejuvenate the brain and alert the senses \\
\hline $11-$ & Detoxify the liver, making body healthier overall, as well as make the cholesterol numbers lower \\
\hline $12-$ & It may help alleviate pimples, acne, and other skin problems \\
\hline $13-$ & It may lower body temperature, and can help relieve the pain \\
\hline $14-$ & $\begin{array}{l}\text { It may help feeling better without the nasty side effects that chemical medicines may have, particularly } \\
\text { prescription }\end{array}$ \\
\hline
\end{tabular}


Table 16. The most important health benefits of chrysanthemum tea

\begin{tabular}{ll}
\hline $1-$ & Anti-inflammatory \\
\hline $2-$ & Increase immune system \\
\hline $3-$ & Strengthens bones \\
\hline $4-$ & Prevents chronic diseases \\
\hline $5-$ & Improve eyesight \\
\hline $6-$ & Increase metabolic rate \\
\hline $7-$ & Maintains cardiovascular health \\
\hline $8-$ & Relaxes nerves \\
\hline $9-$ & Treats cough and cold \\
\hline $10-$ & Detoxifies body \\
\hline
\end{tabular}

Table 17. Samples and origins of the studies Chrysanthemum morifolium tea from China (Wang et al., 2019)

\begin{tabular}{|c|c|c|}
\hline Samples & Company & Origins \\
\hline C. morigolium Gongju & $\begin{array}{l}\text { Beijing Tongrentang Health } \\
\text { Pharmaceutical Industry Co., Ltd }\end{array}$ & Huangshan city, Anhui province \\
\hline C. morifolium Hangbaiju & $\begin{array}{l}\text { Beijing Tongrentang Health } \\
\text { Pharmaceutical Industry Co., Ltd }\end{array}$ & $\begin{array}{c}\text { Tongxiang city, Zhejiang } \\
\text { Province }\end{array}$ \\
\hline C. morifolium Taiju & $\begin{array}{c}\text { Beijing Zhang Yiyuan Jinqiao Tea Co., } \\
\text { Ltd. }\end{array}$ & $\begin{array}{l}\text { Zhongwei city, Ningxia Hui } \\
\text { Autonomous Region }\end{array}$ \\
\hline C. morifolium Boju & $\begin{array}{c}\text { Bozhou Zhongyitang Chinese Medicinal } \\
\text { Materials Sales Co., Ltd. }\end{array}$ & Bozhou city, Anhui Province \\
\hline C. morifolium Chuju & $\begin{array}{c}\text { Anhui Jutai Chuju Herb Science and } \\
\text { Technology Co., Ltd. }\end{array}$ & Chuzhou city, Anhui Province \\
\hline C. morifolium Huangju & $\begin{array}{l}\text { Huangshan Dingxiangwu Ecological } \\
\text { Agriculture Development Co., Ltd. }\end{array}$ & Shangrao city, Jiangxi Province \\
\hline
\end{tabular}

Table 18. Common TCM formulas in which chrysanthemum flowers are used

\begin{tabular}{ll}
\hline $1-$ & For hypertension combine chrysanthemum flowers with dangelions and honeysuckle flowers \\
\hline $2-$ & $\begin{array}{l}\text { For improving vision, relieving tinnitus and headaches combine chrysanthemum flowers with goji } \\
\text { berries }\end{array}$ \\
\hline $3-$ & $\begin{array}{l}\text { For exterior wind-heat with symptoms of headache, colds, sore throat combine chrysanthemum } \\
\text { flowers with wild mint, platycodon roots, and greater burdock fruits }\end{array}$ \\
\hline $4-$ & $\begin{array}{l}\text { For high blood pressure combine chrysanthemum flowers with gambir stems and thorns, cassia } \\
\text { seeds and white peony roots }\end{array}$ \\
\hline $5-$ & $\begin{array}{l}\text { For liver and kidney Yin deficiency combine chrysanthemum flowers with glossy privet fruits and } \\
\text { goji berries }\end{array}$ \\
\hline $6-$ & $\begin{array}{l}\text { For external wind heat with fever, sore throat, chills and red eyes combine chrysanthemum } \\
\text { flowers with mulberry leaves, forsythia fruits, wild mint and platycodon roots }\end{array}$ \\
\hline $7-$ & $\begin{array}{l}\text { For deficient kidney and liver Yin patterns with symptoms such as dizziness, vertigo, blurred } \\
\text { vision, headache and hypertension combine chrysanthemum flowers with heal-all spikes, gambir } \\
\text { stems and thorns and Baikal skullcap roots }\end{array}$ \\
\hline $8-$ & $\begin{array}{l}\text { For wind-heat headache combine chrysanthemum flowers with angelica roots and Szechuan } \\
\text { lovage roots }\end{array}$ \\
\hline $9-$ & $\begin{array}{l}\text { For liver or wind-heat with red and painful eyes combine chrysanthemum flowers with cassia } \\
\text { seeds and mulberry leaves }\end{array}$ \\
\hline
\end{tabular}




\section{Conclusions}

Chrysanthemum is the second most important plants in both ornamental industry and traditional Chinese medicine. Chrysanthemums, often called mums or chrysanths (family Asteraceae, genus Chrysanthemum), are one of the most important crops in the flower industry. Chrysanthemum is both a source of beautification and high medicinal characteristics. In some Eastern cultures, chrysanthemums are also a symbol of good luck, wealth, happiness, and longevity. In Chinese medicine, chrysanthemums are a versatile herb called Ju Hua. In Chinese traditional medicine, Ju Hua is considered to be a fragrant, cool, and light herb. Also, it is used for cooling heat in the liver channel, especially when it manifests as dry, red or painful eyes. It has tremendously diverse in morphologies including flower shapes, sizes, colors and plant architecture. Thirteen important compounds of chrysanthemum flowers are acacetin-7-0-beta-Dglucopyranoside, luteolin, luteolin-7-O-beta-D-glucopyranoside, acaciin, acacetin 7-0(6"-0-alpha-L-rhamnopyranosyl)-beta-sophoroside, 3-0-caffeoylquinic acid, syringaresinol 0-beta-D-glucopyranoside, 5,7-dihydroxychromone, uracil, pgydroxybenzoic acid, 4-0-beta-D-glucopyranosyloxybenzoic acid, boscialin and blumenol A. Some health benefits of chrysanthemums are for allergies, hypertension (high blood pressure0, tightening of the chest, anxiety, skin conditions such as boils, vertigo, eyes that are inflamed, headaches, sore throats, colds and tinnitus. In traditional Chinese medicine, chrysanthemum is a cold herb which helps dispel pathogenic heat, helps the liver, improves eyesight and aids detoxification. In TCM, chrysanthemum tea has been used for varicose veins, atherosclerosis, acne, influenza, sore throat, fever, angina (Chest pain), common cold symptoms, high blood pressure, inflammation, HIV/AIDS, dizziness and type 2 diabetes. Integrative use of modern agriculture and science of traditional Chinese herbs with new technologies will play an important role in sustainable agriculture and food systems. More clinical researches are necessary to uncover the numerous substances and their impacts on chrysanthemum that contribute to public health.

Conflict of interests. No potential conflict of interests was reported by the authors.

\section{REFERENCES}

[1] Bose, T. K., Yadav, L. P., Pal, V. A., Parthasarathy, Das, P. (2003): Commercial Flowers. Vol. II. - Naya Udyog, Kolkata, India.

[2] Carvalho-Zanao, M. P., Zanao Junior, L. A., Barbosa, J. G., Grossi, J. A. S., De A Vila, V. T. (2012): Yield and shelf life of chrysanthemum in response to the silicon application. - Hort. Bras 30(3): 403-408.

[3] Chukki, J., Abinandan, S., Shanthakumar, S. (2018): Chrysanthemum indicum microparticles on removal of hazardous Congo red dye using response surface methodology. - Int. J. Ind. Chem https://doi.org/10.1007/s40090-018-0160-5.

[4] Chung, Y. S., Jun, T., Lee, Y. G., Jung, J. A., Won, S. Y., Hwang, Y. J., Silva, R. R., Choi, S. C., Kim, C. (2018): A genetic linkage map of wild Chrysanthemum species indigenous to Korea and its challenges. - Int. J. Agric. Biol 20: 2708-2716.

[5] Cicek Atikmen, N., Kutuk, C., Karahan, G. (2014): Response of chrysanthemum (Chrysanthemum morifolium) to fresh and exhausted mushroom compost substrates in greenhouse conditions. - Bulletin UASVM Horticulture 71(2): 233-239. 
[6] Cojocariu, A., Chelariu, E. L., Tanase, C. (2018): Adventitious roots development and root system architecture of chrysanthemum cuttings. - J. Plant Develop 25: 91-98.

[7] Datta, S. K. (2013): Chrysanthemum morifolium Ramar. A unique genetic material for breeding. - Sci Cult 7-8: 307-313.

[8] Dong, L., Huang, Z., Liu, D., Zhu, P., Lv, S., Li, N., Mao, H. (2018): Transcriptome analysis of chrysanthemum in responses to white rust. - Sci Hort 233: 421-430.

[9] Imtiaz, M., Khattak, A. M., Ali Khan, M., Jalal, F., Hussain, S., Said, F., Bo, H. (2019): Rapid in-vitro propagation of chrysanthemum morifolium through shoot bud explants. Pak. J. Bot 51(3).

[10] Kalia, R. (2015): Effect of different concentrations of auxins on the regeneration of Chrysanthemum Morif Olium plantlets. - Int. J. Tech. Res. Applic 3(6): 106-107.

[11] Kaur, M., Dahiya, D. S., Kumar, S., Yadav, G., Malik, A. (2018): Appraisal for flower yield and genetic correlation of Chrysanthemum morifolium genotypes in semi-arid Haryana. - J. Pharmacogn Phytochem 7(4): 1267-1272.

[12] Kumar, G., Singh, K. P., Prasad, K. V., Rana, M. K., Namita, Panwark, S. (2014): Genetic diversity analysis of chrysanthemum (Chrysanthemum grandiflorum) cultivars using RAPD markers. - Indian J. Agr. Sci 84(11): 1323-8.

[13] Klie, M., Schie, S., Linde, M., Debener, T. (2014): The type of ploidy of Chrysanthemumis not black or white: a comparison of a molecular approach to published cytological methods. - Front Plant Sci 479: 1-8.

[14] Lee, M. K., van Iersel, M. W. (2008): Sodium chloride effects on growth, morphology, and physiology of chrysanthemum (Chrysanthemum $\times$ morifolium). - HortScience 43(6): 1888-1891.

[15] Liang-Yu, W., Hong-Zhou, G., Xun-Lei, W., Jian-Hui, Y., Jian-Liang, L., Yue-Rong, L. (2010): Analysis of chemical composition of Chrysanthemum indicum flowers by GC/MS and HPLC. - J. Med. Plants Res 4(5): 421-426.

[16] Lin, L., Mao, X., Sun, Y., Rajivgandhi, G., Cui, H. (2019): Antibacterial properties of nanofibers containing chrysanthemum essential oil and their application as beef packaging. - Int. J. Food Microbiol 292: 21-30.

[17] Liu, F., Ong, E. S., Li, S. F. (2013): A green and effective approach for characterization and quality control of chrysanthemum by pressurized hot water extraction in combination with HPLC with UV absorbance detection. - Food Chem 141: 1807-1813.

[18] Liu, L. L., Xiao, Z. B. (2018): Chemical constituents from flowers of Chrysanthemumindicum. - Chinese Traditional and Herbal Drugs 49(22): 5254-5258.

[19] Marongiu, B., Piras, A., Porcedda, S., Porcedda, S., Tuveri, E., Laconi, S., Deidda, D., Maxia, A. (2009): Chemical and biological comparisons on supercritical extracts of Tanacetum cinerariifolium (Trevir) Sch. Bip., with three related species of Chrysanthemums of Sardinia (Italy). - Nat. Prod. Res 23: 190-199.

[20] Michalowska, E., Lema-Ruminska, J. (2018): ISSR markers in the genetic diversity of Chrysanthemum plants derived via somatic embryogenesis. - Zeszyty Problemowe Postepow Nauk Rolniczych 594: 17-25.

[21] Niu, Y., Sun, X., Xiao, Z., Wang, P., Wang, R. (2018): Olfactory impact of terpene alcohol on terpenes aroma expression in Chrysanthemum essential oils. - Molecules 23: 2803.

[22] Ogbaji, P. O., Li, J., Xue, X., Shahrajabian, M. H., Egrinya, E. A. (2018): Impact of biofertilizer or nutrient solution on Spinach (Spinacea Oleracea) growth and yield in some province soils of P. R. China. - Cercetari Agronomice in Moldova 2(174): 43-52.

[23] Prakash, A., Kumar, M., Kumar, A., Kumar, M., Gupta, A., Badal, S. (2018): Performance and flower characterization of chrysanthemum (Dendranthema grandiflora Tzvelev) genotypes under agro-climatic region of western Uttar Pradesh. - Int. J. Chem. Stud 6(5): 1439-1442. 
[24] Saicharan, M., Anitha, V., Kameshwari, L., Srilatha, D. (2017): Seasonal incidence of insect pests of chrysanthemum in Maddur and Palgutta villages of Ranga Reddy district, Telangana. - Int. J. Farm Sci 7(4): 141-143.

[25] Sassi, A. B., Harzallah-Skhiri, N., Bourgougnon, N., Aouni, M. (2008): Antimicrobial activities of four Tunisian Chrysanthemum species. - Indian J. Med. Res. 127: 183-192.

[26] Shahrajabian, M. H., Sun, A., Cheng, Q. (2018): A review of goji berry (Lycium barbarum) in traditional Chinese medicine as a promising organic superfood and superfruit in modern industry. - Academia Journal of Medicinal Plants 6(12): 437-445.

[27] Shahrajabian, M. H., Sun, W., Cheng, Q. (2019a): The power of natural Chinese medicine, ginger and ginseng root in an organic life. - Middle-East J. Sci. Res 27(1): 6471.

[28] Shahrajabian, M. H., Sun, W., Cheng, Q. (2019b): Traditional Chinese medicine and agriculture; organic life and sustainability for future. - GSC Biological and Pharmaceutical Sciences 7(01): 091-095.

[29] Shahrajabian, M. H., Sun, W., Cheng, Q. (2019c): Clinical aspects and health benefits of ginger (Zingiber officinale) in both Traditional Chinese and modern industry. - Acta Agriculturae Scandinavica, Section B-Soil \& Plant Science. DOI: 10.1080/09064710.2019.1606930.

[30] Shahrajabian, M. H., Khoshkharam, M., Sun, W., Cheng, Q. (2019d): The effect of pretreatment factors on seed germination and seedling growth of (Pimpinella anisum L.). - Middle East J. Sci. Res 5(1): 86-93.

[31] Shahrajabian, M. H., Sun, W., Cheng, Q. (2019e): A review of ginseng species in different regions as a multipurpose herb in traditional Chinese medicine, modern herbology and pharmacological science. - J. Med. Plants. Stud 13(10): 213-226.

[32] Shahrajabian, M. H., Sun, W., Cheng, Q. (2019f): Modern pharmacological actions of Longan fruits and their usages in traditional herbal remedies. - J. Med. Plants. Stud 7(4): 179-185.

[33] Shahrajabian, M. H., Khoshkharam, M., Zandi, P., Sun, W., Cheng, Q. (2020): The influence of temperatures on germination and seedling growth of pyrethrum (Tanacetum Cineraiifolium) under drought stress. - International Journal of Advanced Biological and Biomedical Research 8(1): 9-39 (in press).

[34] Shrestha, S., Satyal, P., Pandit, G., Setzer, W. N. (2014): Chemical composition of the essential oil from the aerial parts of Chrysanthemum cinerariifolium growing in Nepal. Am. J. Essent. Oi. Nat 2(2): 01-03.

[35] Soleymani, A., Shahrajabian, M. H. (2012): Response of different cultivars of fennel (Foeniculum vulgare) to irrigation and planting dates in Isfahan, Iran. - Res Crops 13(2): 656-660.

[36] Soleymani, A., Shahrajabian, M. H. (2018): Changes in germination and seedling growth different cultivars of cumin to drought stress. - Cercetari Agronomice in Moldova 1(173): 91-100.

[37] Sukmawani, R., Haerumank, M., Sulistyowati, L., Perdana, T. (2015): Sustainable development model of Chrysanthemum agribusiness. - Int. J. Agric. Sci. Res 5(1): 11-18.

[38] Sun, C. Q., Huang, Z. Z., Wang, Y. L. et al. (2011): Overcoming prefertilization barriers in the wide cross between Chrysanthemum grandiflorum (Ramat.) Kitmaura and $C$. nankingense (Nakai) Tzvel. By using special pollination techniques. - Euphytica 178(2): 195-202.

[39] Teixeria da Silva. (2003): Chrysanthemum organogenesis through think cell layer technology and plant growth regulator control. - Asian J. Plant Sci 2(6): 505-514.

[40] Wang, J., Xiao, H. (2013): Discrimination of different white chrysanthemum by electronic tongue. - J. Food. Sci. Technol 50: 986-992.

[41] Wang, F., Zhang, F. J., Chen, F. D., Fang, W. M., Teng, N. J. (2014): Identification of Chrysanthemum (Chrysanthemum morifolium) self-incompatibility. - Hindawi Article ID 625658 . 
[42] Wang, Y., Xu, Z., Huang, Y., Wen, X., Wu, Y., Zhao, Y., Ni, Y. (2018): Extraction, purification and hydrolysis behavior of apigenin-7-O-glucoside from Chrysanthemum morridolium tea. - Molecules 23: 2933.

[43] Wang, Y., Xu, Z., Wu, Y., Li, M., Pang, S., Liang, Z., Ni, Y. (2019): Effect of weakly basic conditions on the separation and purification of flavonoids and glycosides from Chrysanthemum morifolium tea. - Molecules 24: 297.

[44] Yang, P. F., Yang, Y. N., He, C. Y., Chen, Z. F., Yuan, Q. S., Zhao, S. C., Fu, Y. F., Zhang, P. C., Mao. D. B. (2019): New caffeoylquinic acid derivatives and flavanone glycoside from the flowers of Chrysanthemum morifolium and their bioactivities. Molecules 24: 850.

[45] Yasemin, S., Koksal, N., Ozkaya, A., Yener, M. (2017): Growth and physiological responses of Chrysanthemum paludosum under salinity stress. - J. Biol. Environ. Sci 11(32): 59-66.

[46] Yu, Y., Zhu, C., Wang, S., Song, W., Yang, Y., Shi, J. (2013): Homosecoiridoid alkaloids with amino acid units from the flower buds of Lonicera japonica. - J. Nat. Prod 76(12): 2226-2233. 